\title{
A Study to Determine the Prevalence of Premenstrual Syndrome in Young Females
}

\author{
Apeksha Vaishnavi Thodupunuri' ${ }^{1}$, Suwaibah Fatima Samer'², Mohammed Abrar Hassan ${ }^{3}$ \\ ${ }^{1}$ MBBS Student, Bhaskar Medical College, ${ }^{2}$ Assistant Professor of Physiology Bhaskar Medical College, \\ ${ }^{3}$ Professor and HOD of Physiology Bhaskar Medical College
}

\begin{abstract}
Introduction: Premenstrual syndrome can be broadly classified as any constellation of psychological and physical symptoms that recur regularly in the luteal phase of the menstrual cycle; remit for at least 1 week in the follicular phase and cause distress and functional impairment. In order to be clinically significant, the symptoms should be of at least moderate intensity and cause functional impairment. Women with premenstrual dysphoric disorder report impaired social adjustment and reduced perceived quality of life, which are at their worst during the luteal phase. Premenstrual syndrome differs from depression and physical symptoms such as bloating, mastalgia and headache. The most common reported psychological symptom is irritability rather than depressed mood.
\end{abstract}

Materials and Method: A cross sectional study has been conducted in Bhaskar medical college from June 2018 to July 2018 sponsored by ICMR,on 150 subjects .Data of 100 was collected on a 29 itemed shortened premenstrual assessment form based on Moos Menstrual Distress Questionnaire. The study protocol and the Questionnaire were presented to the Institutional Ethics Committee (IEC).

Results: The results obtained are Based on symptomatology, (i) Percentage of symptoms of PMS less than $50 \%$ and Above $50 \%$ criteria was considered for statistical analysis.

Discussion: Many women have premenstrual cyclic symptoms of psychological or physical nature and sometimes these limit their functional capacity, While PMS is linked to the luteal phase, the cause of PMS is not clear, but several factors may be involved. Changes in hormones during the menstrual cycle seem to be an important factor.

Conclusion: As PMS is becoming problem in young girls hampering their life involving physical and psychic symptoms.

Keywords: Premenstrual syndrome(PMS), Premenstrual dysphoric disorder, Premenstrual distress, Moos Menstrual Distress Questionnaire.

\section{Introduction}

Premenstrual syndrome can be broadly classified as any constellation of psychological and physical

\section{Corresponding Author:}

\section{Dr. Mohammed Abrar Hassan}

Address: 10-2-164/D, 37, West Marredpally, Road number: 2, Opposite Johnson Grammar School, Secunderabad, 500026 Hyderabad, India symptoms that recur regularly in the luteal phase of the menstrual cycle; remit for at least 1 week in the follicular phase and cause distress and functional impairment. It is generally accepted that, in order to be clinically significant, the symptoms should be of at least moderate intensity and cause functional impairment. Severe symptoms that are predominantly dysphoric and cause severe impairment are referred to as premenstrual dysphoric disorder ${ }^{5}$. The premenstrual syndrome has been described as the commonest endocrine disorder .Fluhmann ${ }^{8}$ defined premenstrual tension as including 
adverse signs and symptoms of a general systemic nature which manifest themselves rhythmically during the later premenstrual or early menstrual phases. He also stated that there is considerable difficulty in describing premenstrual tension as an entity because of lack of adequate information based on large groups of normal women a need exists for detailed investigation. The development of standard method for collecting cross sectional and longitudinal information on menstrual cycle symptoms would appear to be potentially useful both for comparing estimates of the prevalence and severity of symptoms in various populations and for more careful study of the psychological and biochemical correlates of different types of menstrual and premenstrual distress. ${ }^{7}$

Christer and Caplan $^{5}$ described premenstrual syndrome as a form of social control and victim blame. Women with premenstrual dysphoric disorder recorded in their medical record might be seen as unfit mothers in child custody cases and as unsuitable candidates for positions of authority or political office, thus leading to increased bias and discrimination against women.

Women with premenstrual dysphoric disorder report impaired social adjustment and reduced perceived quality of life, which, unsurprisingly, are at their worst during the luteal phase. Premenstrual syndrome differs from depression in its wider symptom profile, including physical symptoms such as bloating, mastalgia and headache. The most common reported psychological symptom is irritability rather than depressed mood. Women with mood disorders (unipolar or bipolar) or anxiety disorders may experience significant mood changes in the premenstrual period

\section{Materials and Method}

A cross sectional study has been conducted in Bhaskar medical college from June 2018 to July 2018 sponser by ICMR on 150 female subjects out of which 100 were selected for analysis.

Inclusion Criteria: Unmarried girls who were having regular menstrual cycles for the last 6 months in the age group of 18-24 years with or without PMS.

Exclusion Criteria: They were excluded if they had any psychological or medical disorder such as thyroid, PCOD or if they had irregular menstrual cycles in the last 6 months, having addictions like smoking drinking alcohol or drugs or those taking treatment for any other diseases.
Data was collected on a 29 itemed shortened premenstrual assessment form based on Moos Menstrual Distress Questionnaire. ${ }^{6}$ Psychological, physical, behavioural symptoms were studied for 2 cycles. The number of symptoms present was noted on each day of luteal phase starting from day 14 of the same cycle, assuming total cycle length to be 28 days ${ }^{1}$. The study protocol and the Questionnaire were presented to the Institutional Ethics Committee (IEC) first and prior approval obtained and consent was also taken from the participants and then given to them to be filled prospectively over 2 cycles.

An ICD-10 symptom checklist for PMS was used to identify girls with PMS. The ICD- criteria for PMS includes seven symptoms: minor physiological discomfort, bloating or weight gain, breast tenderness, muscular tension, aches and pains, poor concentration and changes in appetite. Only one of these symptoms is required for diagnosis. Symptoms must be restricted to the luteal phase of menstrual cycle and cease with commencement of menstrual flow ${ }^{1}$

\section{Results}

Based on symptomatology, the results obtained are:

(i) Percentage of symptoms less than $50 \%$ : 1) Confusion-49\%; 2) Depressed feeling-44\%; 3) Guilty-28\%; 4) Hopeless feeling-39\%; 5) Loneliness-32\%; 6) Lowered selfesteem-36\%; 7) Disinterest in life-36\%; 8) Abdominal bloating-36\%; 9) Absent mindedness-39\%; 10) Binge eating-40\%; 11) Breast tenderness-33\%;12) Constipation- $21 \%$; 13) Oedema-2\%; 14) Fainting-20\%; 15) Insomnia-25\%; 16) Weightgain-35\%; 17) Nausea-24\%; 18) Prone to violent outbursts-34\%; 19) Abstinence from work-35\%; 20) Personality Change-21\%

Above 50\% criteria was considered for following symptoms in Premenstrual Syndrome:

1) Angered easily-79\%; 2) Anxiety-61\%; 3) Irritability-89\%; 4) Moodiness-71\%; 5) Stressful Feeling-84\%; 6) Back Pain-78\%; 7) General Body Discomfort-75\%; 8) Headache-50\%; 9) Fatigue-67\%.

The data was also subjected to a pre-validated Moos Menstrual Distress Questionnaire which is based on 8 broad parameters, each one include 3-4 sub parameters and specific points are given. 


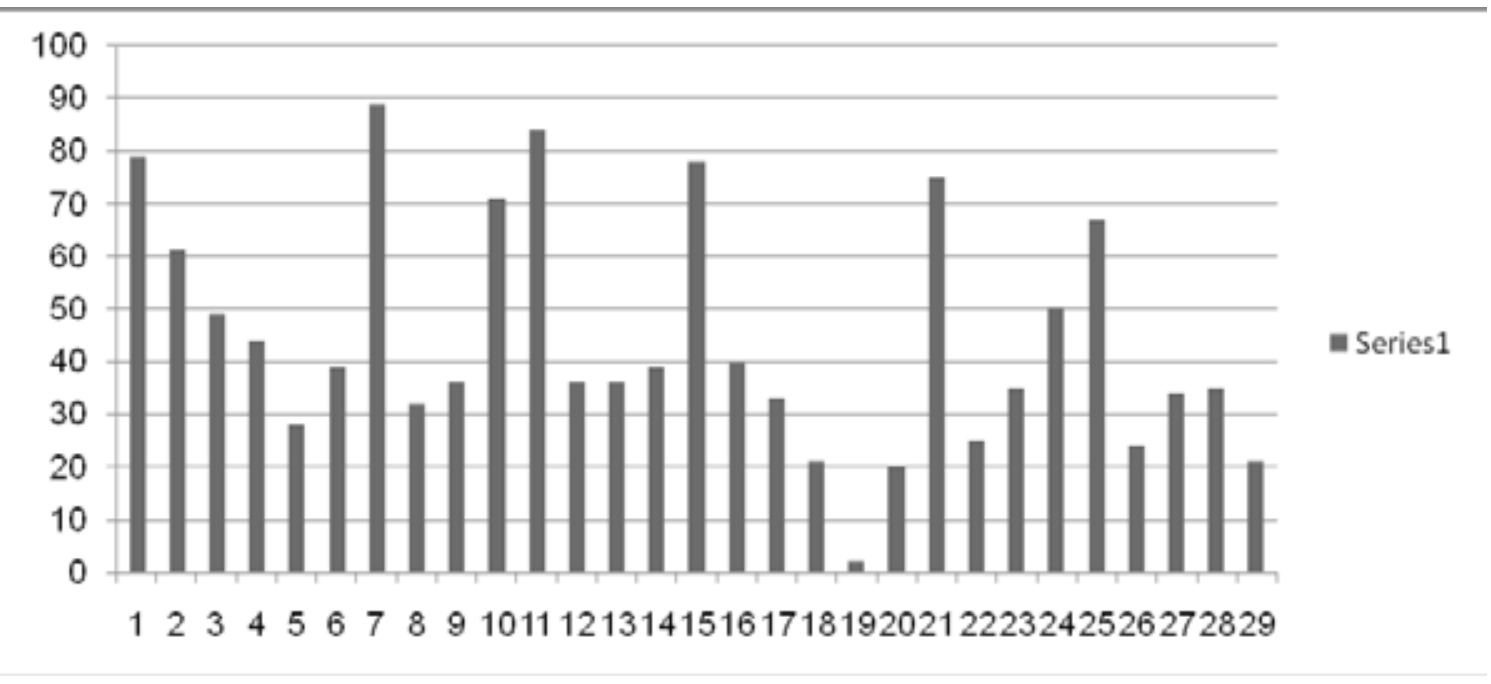

X-Axis indicates all the 29 symptoms, Y-Axis indicates the percentage of subjects with those symptoms.

The total score obtained in each group: A) Pain=114; B) Concentration=178; C) Behavioural change $=88$; D) Autonomic Reaction $=94$; E) Water retention=75; F) Negative affect=221; G) Arousal=123; H) Control=191

\section{Discussion}

Many women have premenstrual cyclic symptoms of psychological or physical nature and sometimes these limit their functional capacity. ${ }^{3}$

High prevalence of PMS are Estradiol and progesterone level in the luteal phase which affects the neurotransmitters' serotonin and GABA, increased carbohydrate intolerance in the luteal phase. Pyridoxine deficiency- this vitamin plays a role in oestrogen synthesis, dopamine and serotonin production.it is also associated withincreased production of vasopressin, aldosterone, prolactin and systemic prostaglandins adversely affecting renal function and contributing to fluid retention and bloating. Fluctuation is the opiate peptide concentration affecting endorphin levels. It is not clear whether PMS is an abnormal response to normal hormone fluctuations or whether hormonal imbalances could lead to premenstrual syndrome. ${ }^{2}$

PMS completely resolves at menopause. Eating 4 to 6 smaller meals per day during the premenstrual period may help reduce symptoms and food craving. Avoidance of salt, caffeine, alcohol, chocolate or simple carbohydrate may alleviate symptoms the benefits of exercise include physical improvements as well as stress reductions. ${ }^{1}$
Ovulation suppressants like Gonadotropin releasing hormone (GNRH) agonists lead to decreased follicle stimulating hormone (FSH) and Luteinizing hormone (LH) release from pituitary resulting in decreased oestrogen and progesterone levels. Use of progesterone in the luteal phase has been one of the strategic measures to treat PMS which holds well even today. Recently selective serotonergic reuptake inhibitors [SSRI] have gained greater importance in the management of PMS. ${ }^{4}$

While Premenstrual Syndrome is linked to the luteal phase, the cause of Premenstrual Syndrome is not clear, but several factors may be involved. Changes in hormones during the menstrual cycle seem to be an important factor; changing hormone levels affect some women more than others.

Girls using psychotropic were excluded ensuring an untreated sample. ${ }^{6}$ Chemical changes in the brain, stress and emotional problems such as depression do not seem to cause PMS but they may make it worse. Low levels of vitamins and minerals, high sodium, alcohol and or caffeine can exacerbate symptoms such as water retention and bloating. Magnesium and Calcium deficiencies are postulated as nutritional cause of PMS; studies evaluating supplementation show improvement in physical and emotional symptoms. ${ }^{1}$

\section{Conclusion}

As Premenstrual Syndrome is becoming a common day to day problem in young girls hampering their life involving physical and psychic symptoms. Maximum participants do not seek medical advice and take selftreatment. 
Doctors should adopt comprehension measures to reduce its incidence and improve the quality of life in affected. A significant relation was present between Premenstrual Syndrome and psychiatric involvement and dysmenorrhoea therefore comprehensive efforts should be made by healthcare providers to screen and manage symptoms including dysmenorrhoea efficiently, thereby improving daily functioning, academic performance and professional skills of the students. Irrespective of the age, literacy and socio economic status, most of the women tend to suffer with PMS, which may be understood by them or they may be ignorant of it. As Premenstrual Syndrome is becoming a common day to day problem in young girls hampering their life involving physical and psychic symptoms, in a country like ours often it is even taken as a stigma to discuss the issues related to menstrual cycle.

\section{Conflict of Interest: Nil}

\section{Source of Funding: Self}

Ethics Committee: Certificate

\section{References}

1. Silpa Gantela, Srilatha Choppara. Severity and Frequency of Premenstrual Syndrome in college girls aged 18-25 years. Journal of Evolution of Medical and Dental Sciences 2015; Vol.4, Issue 87, October 29; Page: 15228-15232, DOI: 10. 14260/ jemds/2015/2164
2. Kavitha. P, Shanmughavadivu. R; A Study on the prevalence of Pre Menstrual Syndrome and its relationship with Anthropometric Indices. TJPRC: International Journal of Pharmacology and Physiology; Vol. 1, Issue 1, Jun 2015, 27-32

3. Shruti Brahmabhatt, B.M Sattigeri, Heena Shah, Ashok Kumar, Devang Parikh; A prospective survey study on Premenstrual Syndrome in young and middle aged women with an emphasis on its management; International Journal of Research in Medical Sciences; 2013 May; Vol 1; Issue 2: 69-72

4. J.V. Joshi, S.N. Pandey, P.Galvankar and J.A. Gogate; Prevalence of Premenstrual symptoms: Preliminary analysis and brief review of management strategies; Journal of Mid-Life Health; 2010 Jan-Jun; 1(1): 30-34

5. Carol. A. Henshaw; PMS: Diagnosis, aetiology, assessment and management; Advances in Psychiatric Treatment Feb 2007, 13(2) 139-146; DOI: 10.1192/apt.bp.106.003392

6. Samia Tabassum, Bilquis Afridi, Zahid Aman, Wajeeha Tabassum, Rizwana Durrani; JPak Med Assoc; Vol. 55, No. 12, December 2005; 546-549

7. Rudolf. H. Moos; The development of a menstrual Distress questionnaire; Vol XX, No.6, 1968

8. Fluhmann, C.F. Management of Menstrual Disorders. Saunders, Philadelphia, 1956. 